\title{
Thinking Like Disney: Supporting the Disney Method Using Ambient Feedback Based on Group Performance
}

\author{
Sarah Tausch $^{1(\bowtie)}$, Fabius Steinberger ${ }^{1,2}$, and Heinrich Hußmann ${ }^{1}$ \\ ${ }^{1}$ Media Informatics Group, University of Munich (LMU), Munich, Germany \\ \{sarah. tausch, hussmann\}@ifi.lmu.de, \\ fabius. steinberger@qut. edu. au \\ ${ }^{2}$ Urban Informatics Research Lab, Queensland University of Technology, \\ Brisbane, Australia
}

\begin{abstract}
The Disney method is a collaborative creativity technique that uses three roles - dreamer, realist and critic - to facilitate the consideration of different perspectives on a topic. Especially for novices it is important to obtain guidance in applying this method. One way is providing groups with a trained moderator. However, feedback about the group's behavior might interrupt the flow of the idea finding process. We built and evaluated a system that provides ambient feedback to a group about the distribution of their statements among the three roles. Our preliminary field study indicates that groups supported by the system contribute more and roles are used in a more balanced way while the visualization does not disrupt the group work.
\end{abstract}

Keywords: Collaborative creativity $\cdot$ Disney method $\cdot$ Feedback $\cdot$ Group mirror $\cdot$ Tabletop display

\section{Introduction}

Collaborative creativity (also called social creativity) is a common way to find novel ideas and can be used as an important part of problem solving. Fischer [1] describes this concept as interplay of spatial, temporal, conceptual and technological aspects. These dimensions can be interpreted as barriers but at the same time introduce opportunities for the design of socio-technical systems.

Large displays that blend into the environment are one way of supporting collaborative processes, realizing the concept of ubiquitous computing. Displaying ambient information on such screens during group work shifts the focus from human-computer interaction to technologically mediated human-human interaction. In particular, group mirrors can facilitate this approach. These are systems that support collaboration by reflecting certain aspects of group work to the group [2], for example by visualizing speaking times on a peripheral display.

Our approach investigates the combination of creativity and group mirrors. As such, we aim to create unobtrusive, ambient support for creative group work. Previous research has mainly focused on the influence of feedback about individual performance 
on collaborative processes. Despite several positive effects, this kind of feedback can lead to social pressure or frustration among group members [3, 4]. In contrast to previous research, our system visualizes group performance rather than individual performance. Specifically, we intend to investigate if this kind of feedback supports performance while being less disruptive and reducing social pressure.

We further explored if our system supports a particular creativity technique, the Disney method. This method uses three different roles - dreamer, realist and critic - to allow various perspectives on one topic. In a preliminary field study we investigated if our system improves performance without causing disruptions and if all roles are equally made use of, which is a key element of the Disney method.

\section{Related Work}

There is a tremendous amount of literature on creativity, its background and computer support for creativity (cf. overview by Sternberg [5]). We will restrain on discussing creativity support for co-located collaboration. Most tools supporting co-located creative group work use large interactive displays integrated into tables and walls. Hilliges et al. [6] for instance combined both display types to build a socio-technical environment for brainstorming. WordPlay [7] uses speech recognition and a multi-touch keyboard for the input of ideas on a table. The influence of feedback during brainstorming has been investigated for instance by Paulus and Dzindolet [8] who could show that information about performance of other groups can influence participation behavior.

Balancing participation is the main goal of several group mirrors. The Meeting Mediator [9] and a tool of Schiavo et al. [4] use private displays to visualize feedback about visual attention from and to others. Most group mirrors however use large displays, such as Reflect [3], a system that displays speaking times in form of colored LEDS integrated into a table. The Second Messenger [10] and the Conversation Clock [11] are visualizations of speaking times and speaking turns, while the latter is a bit more complex as it includes the history and indicates overlapping speech. Finally, Groupgarden [12] is a metaphorical visualization supporting brainstorming with different designs for table and wall displays.

\section{Using the Disney Method for Evaluating App Ideas}

Dilts [13] describes a collaborative creativity technique called the Disney method that is based on Walt Disney's way of working and thinking. He assumes that the success of Disney originates from using three conceptual positions. This can help group members to think both about both novel ideas as well as critical aspects.

In our study, conducted in a university course for app development, we explained the roles of the Disney method using examples to help teams in the development and evaluation of app ideas. In the following, we will describe the original interpretation of the roles as well as the way we used them. Notably, all roles were used at the same time to preserve the natural flow of the discussion rather than establishing constraints. 
Dreamer. Originally, the dreamer's aim is to produce new ideas and goals. Given a task or challenge, it answers the question "what" and provides a vision. In our use case, the dreamer puts forward ideas regarding the design of the user interface and user experience as well as potentially new features.

Realist. Originally, the realist aims at concretizing the ideas of the dreamer. It answers the question "how". For our use case, the realist evaluates the ideas of the dreamer in respect of feasibility, originality, and significance.

Critic. Originally, the critic identifies issues and addresses constraints. It answers the question "why". For our use case, the critic challenges the concepts and detects errors.

\section{Design and Implementation}

A challenge for groups using the Disney method is remembering the roles and integrating them into the creative process. In particular, this is difficult for novices. Furthermore, participants often shy away from the critic role, especially when group members know each other and have to challenge their colleagues' concepts and ideas. We considered these obstacles in the design of our group mirror application.

Four aspects were most important throughout designing the group mirror: (1) the information the system reflects to the group; (2) the visualization; (3) the colors that are used in the visualization; and (4) the placement of the group mirror.

Information. The key element of the Disney method is the use of different roles. Accordingly, the main goal of the group mirror is to encourage groups in using these roles in a fairly balanced way. To support this, the system displays how many statements by a certain role have been made. Implicitly, the overall amount of contributions is visible as well. Displaying the distribution of roles instead of the amount of contributions of individual participants shifts the focus from comparing individual performance to emphasizing team performance. The rationale behind this design decision was to avoid putting pressure on individuals.

Visualization. The visualization consists of three circles, each representing one role. To allow everyone a good view regardless of angle or seating position, we used circles which can easily be compared in size from every position around the table. At the beginning of the discussion, the three circles are equally small. For each new contribution, a small circle moves from the edges of the display to the middle and joins one of the circles in the center (see Fig. 1, left and Fig. 2). Using a combination of simple, colored shapes to visualize the amount of contributions resembles the information decoration approach [14], as it balances aesthetical and informational quality.

Colors. The three roles are represented with colors. Green represents the dreamer, yellow the realist and red the critic. Dilts did not assign colors to the roles, however, using colors makes it easier to perceive information on a peripheral display. Additionally, the circles are labelled with the role names. On the one hand, this assisted participants in remembering the roles and, on the other hand, it made the group mirror accessible for color-blind participants. 

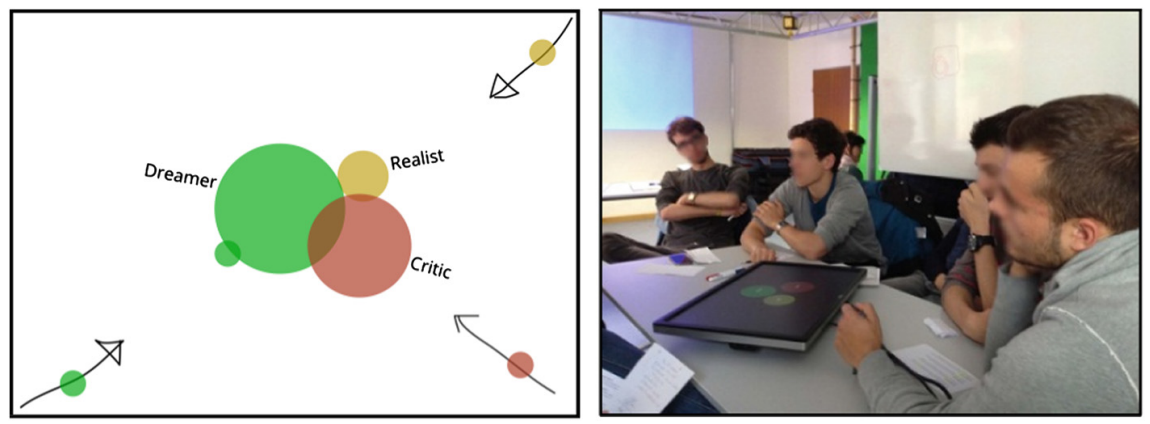

Fig. 1. Left: This sketch shows that with each contribution, a small circle moves to the center and joins the circle of its corresponding color. Right: The group sat around the group mirror that was positioned horizontally on the table.
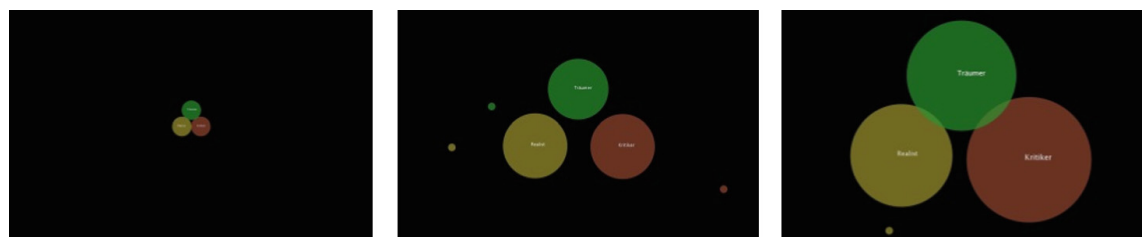

Fig. 2. These screenshots of the group mirror application show (a) how the visualization looks at the start of the group discussion, (b) how a balanced role distribution might look like, and (c) how the visualization could look like at the end of a session.

Placement. Collaboration is a complex task and nonverbal communication such as eye contact, gestures and body language help group members to effectively communicate. Our group mirror therefore follows a calm technology approach [15]. It is designed to stay in the periphery of the attention and to provide feedback to the group in an unobtrusive way to not disrupt communication and to keep the naturalness of the creative group work. We decided to position the group mirror display horizontally on a table (see Fig. 1, right) by lying a 24 in. display flat on a table. In a previous study, Tausch et al. [12] compared a wall version with a table version. Results indicate that feedback on a wall produced less pressure. However, participants perceived the feedback on the table to support collaboration and communication better than the visualization on the wall. As our goal was to accommodate for a natural communication flow, we chose a tabletop setting.

The group mirror application was implemented using Processing and can therefore be executed on Mac OS X, Windows and Linux. In our study, the application ran in full screen mode on an external monitor. The visualization can be controlled via keyboard input. The keys A, S and D signify the three Disney roles. When one of these keys is pressed, the circle of its corresponding role will increase in size. In our experiment, a person who was not participating in the discussion controlled the system. 


\section{Description and Results of a Preliminary Field Study}

We conducted a preliminary field study to investigate the effects of our group mirror on the creative process. Our hypotheses are: (1) The amount of contributions is higher when using the group mirror; (2) the amount of statements by the three roles is more evenly balanced when the group mirror is used; (3) the group mirror does not disrupt the group discussion; and (4) the group mirror does not add additional social pressure.

\subsection{Participants}

16 participants took part in the study. All participants were media informatics students between 20 and 25 years, 5 were female. They were all participants of a course on iOS app development. Most of the participants were novices to the Disney method, only one person knew the method before the study.

\subsection{Method and Procedure}

We chose to conduct a field study instead of a lab study, on the one hand, because group mirrors have mostly been studied in lab environments to date and, on the other hand, to increase external validity [16]. The study was conducted using the Wizard of $\mathrm{Oz}$ technique, meaning that a person sitting next to the groups classified the contributions of the participants and operated the system while the group was not aware of it.

The study took part as an intermediate step of the practical course. Beforehand, participants had developed app ideas in groups of four over a period of three weeks. During the study, each group presented their app idea. After each presentation, the Disney method was used to discuss one app idea for $10 \mathrm{~min}$. As such, four discussions were conducted in total. Participants were divided into two groups, one with support of the group mirror, one without, called baseline in the following. In each of these two groups, six students discussed the idea.

The team that had just presented their idea did not take part in the discussion but listened and took notes. The combinations of participants within the two groups were changed after every discussion to reduce the influence of group dynamics and opinion leaders. All sessions were audio- and video-recorded. Questionnaires were handed out after each session, containing 5-point Likert scales. Results are reported using three categories; 1 and 2: disagreement; 3: neutral; 4: agreement.

\subsection{Results}

We evaluated the study based on application logs, video observations and questionnaire responses. In the following, we will report our results with respect to the previously raised hypotheses.

Quantity of Contributions. In the condition that included usage of the group mirror, participants voiced more contributions (132 in total) than without group mirror (79 in 

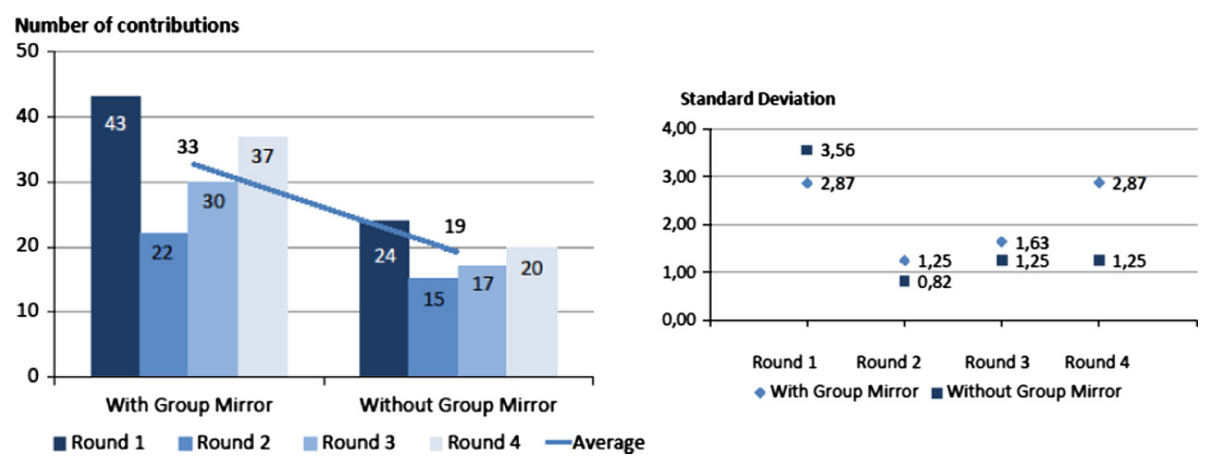

Fig. 3. Left: The number of contributions was higher with group mirror than without. Right: The standard deviation of the different Disney roles was lower in round 1 and higher in round 2, 3 and 4 with group mirror.

total). On average, 33 contributions ( $\mathrm{SD}=9.055)$ were stated with and 19 statements $(\mathrm{SD}=3.916$ ) without visual feedback (see Fig. 3, left). A dependent t-test shows a significant difference $(\mathrm{p}=0.013)$.

Role Distributions. We evaluated how evenly the statements of the three Disney roles were balanced for each session. Specifically, we compared the distributions of the number of statements in each role from their mean. This basically means that we calculated the standard deviation. In three of the four rounds, the standard deviation was lower without group mirror (see Fig. 3, right), however, without a significant difference. It has to be considered, though, that the number of contributions was higher with group mirror and therefore a higher standard deviation is to be expected.

Looking at the distribution of the roles over all rounds, we can see that with group mirror, $33 \%$ of all contributions were dreamer contributions, $39 \%$ realists and $28 \%$ critics. Without group mirror there were $39 \%$ contributions using the role of the dreamer, $36 \%$ realists and $25 \%$ critics. The absolute numbers are shown in Fig. 4. This suggests that the use of the seemingly more difficult roles of the realist and the critic have been particularly supported by the group mirror.
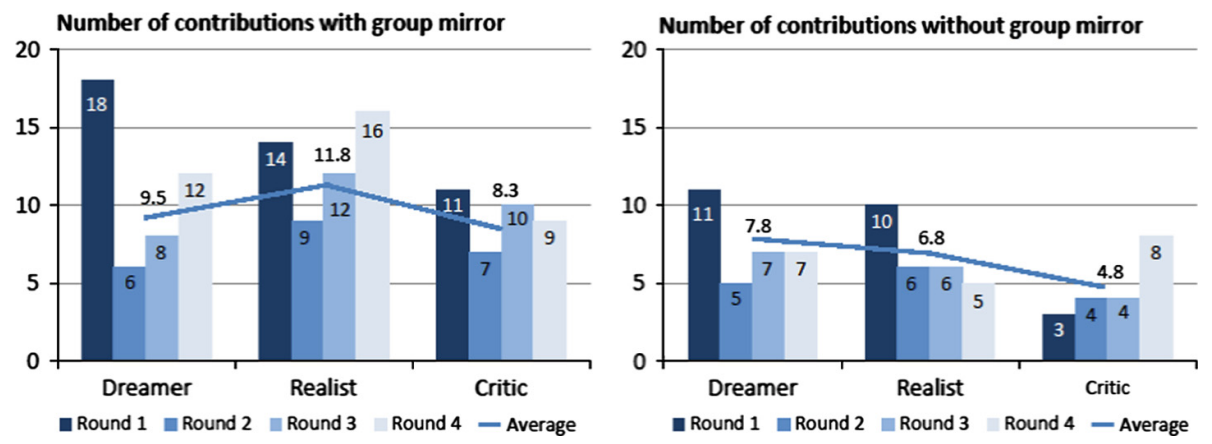

Fig. 4. The number of contributions is higher for all Disney roles with group mirror. 
Disruption. Results of the questionnaires indicate that participants did not perceive the group mirror as disrupting. On a 5-point Likert scale, 15 participants did not agree when asked if the display disrupted the discussion, only one person was neutral regarding this aspect. When asked if participants could follow the discussion and the display, 13 participants agreed, while 3 were neutral. We furthermore asked after both conditions if participants were able to focus on the discussion and whether they felt disrupted. The answers to these questions only differed minimally. In both conditions, 15 participants agreed that they were able to focus with group mirror, one felt neutral, whereas in the baseline one did not agree. In general, participants did not feel distracted a lot, neither with group mirror (13 disagreed, 2 neutral, 1 agreed) nor without (14 disagreed, 2 neutral, 2 agreed).

Social Pressure and Stress. We further asked participants if they felt stressed. Results indicate that people did not perceive themselves as stressed, neither with group mirror (13 disagreed, 2 neutral, 1 abstention) nor without (16 disagreed). When asked about feeling observed, people disagreed or were neutral, both with group mirror (11 disagreed, 5 neutral) and in the baseline (13 disagreed, 3 neutral). When asked if participants perceived others as opponents, answers differed between the group mirror condition (13 disagreed, 1 neutral, 2 agreed) and the baseline (13 disagreed, 3 neutral).

Lastly, we asked participants which condition they preferred. 14 stated that they liked the session with group mirror more, one participant was indifferent and one preferred the discussion without group mirror.

\subsection{Discussion of Results}

We could observe that the distribution of ideas of the three Disney roles differed between both conditions. In the baseline, the role of the critic was used the least. This might be imputable to the fact that participants knew each other and their task was to challenge their colleagues' ideas. The group mirror does not provide any guidance, for example by giving explicit advice to the group. In contrary, it simply shows how often the different roles have been used and thereby implicitly suggests to use the roles in an equilibrated way. Yet, the group mirror had an influence on role distribution and could for instance achieve that the underrepresented role of the critic was used more often than without group mirror.

\section{Conclusion}

In this paper we presented a novel group mirror supporting collaborative creativity and a preliminary field study investigating the effects of the system, providing a basis for next iterations of the group mirror. While designing this group mirror, we aimed to support creativity through subtle and unobtrusive feedback. The approach we chose was to provide feedback about the group performance rather than comparing individual performance. The results of our preliminary study indicate that this type of feedback has a positive impact on the participants' motivation. The overall number of contributions was increased while negative effects such as disruption or stress did not increase. For future work it could be interesting to evaluate the relations to the other 
group members in more details, as our results indicate a difference between both conditions in this regard.

We furthermore have been exploring an interactive tool supporting the Disney method. This will allow us to investigate the differences between an interactive system with more explicit guidance with the subtle and unobtrusive group mirror presented in this paper. As a next step, we plan to compare a group mirror showing individual performance with a visualization that only reflects the group's performance. The idea is to explore if this feedback system can also support other collaborative processes, for example a balanced contribution of all participants.

\section{References}

1. Fischer, G.: Social creativity: turning barriers into opportunities for collaborative design. In: Proceedings of the Eighth Conference on Participatory Design: Artful Integration: Interweaving Media, Materials and Practices, pp. 152-161. ACM (2004)

2. Jermann, P., Soller, A., Muehlenbrock, M.: From mirroring to guiding: a review of state of the art technology for supporting collaborative learning. In: European Conference on Computer-Supported Collaborative Learning, pp. 324-331 (2001)

3. Bachour, K., Kaplan, F., Dillenbourg, P.: An interactive table for supporting participation balance in face-to-face collaborative learning. Learn. Technol. 3, 203-213 (2010)

4. Schiavo, G., Cappelletti, A., Mencarini, E., Stock, O., Zancanaro, M.: Overt or subtle? Supporting group conversations with automatically targeted directives. In: Proceedings of IUI, pp. 225-234. ACM (2014)

5. Sternberg, R.J.: Handbook of Creativity. Cambridge University Press, Cambridge (1999)

6. Hilliges, O., Terrenghi, L., Boring, S., Kim, D., Richter, H., Butz, A.: Designing for collaborative creative problem solving. In: Proceedings of C\&C, pp. 137-146. ACM (2007)

7. Hunter, S., Maes, P.: WordPlay: a table-top interface for collaborative brainstorming and decision making. In: Proceedings of ITS, pp. 2-5 (2008)

8. Paulus, P.B., Dzindolet, M.T.: Social influence processes in group brainstorming. J. Pers. Soc. Psychol. 64, 575-586 (1993)

9. Kim, T., Chang, A., Holland, L., Pentland, A.: Meeting mediator: enhancing group collaboration using sociometric feedback. In: Proceedings of CSCW, pp. 457-466. ACM (2008)

10. DiMicco, J.M., Pandolfo, A., Bender, W.: Influencing group participation with a shared display. In: Proceedings of CSCW, pp. 614-623. ACM (2004)

11. Bergstrom, T., Tony, B., Karrie, K.: Conversation clock: visualizing audio patterns in co-located groups. In: Proceedings of HICSS, pp. 78-87 (2007)

12. Tausch, S., Hausen, D., Kosan, I., Raltchev, A., Hussmann, H.: Groupgarden: supporting brainstorming through a metaphorical group mirror on table or wall. In: Proceedings of NordiCHI, pp. 541-550. ACM (2014)

13. Dilts, R.: Strategies of Genius. Meta Publications, Capitola (1995)

14. Eggen, B., Van Mensvoort, K.: Making sense of what is going on "around": designing environmental awareness information displays. In: Markopoulos, P., De Ruyter, B., Mackay, W. (eds.) Awareness Systems, pp. 99-124. Springer, London (2009)

15. Weiser, M., Brown, J.S.: Designing calm technology. PowerGrid J. 1, 75-85 (1996)

16. Rogers, Y.: Interaction design gone wild: striving for wild theory. Interactions 18, 58-62 (2011) 